\title{
Top Quark Mass Effects in Higgs Boson Production at Four-Loop Order: Virtual Corrections
}

\author{
Joshua Davies, ${ }^{*}$ Florian Herren $\odot{ }^{\dagger}{ }^{\text {and Matthias Steinhauser }}{ }^{\ddagger}$ \\ Institut für Theoretische Teilchenphysik, Karlsruhe Institute of Technology (KIT), 76128 Karlsruhe, Germany
}

(Received 29 November 2019; accepted 3 March 2020; published 20 March 2020)

\begin{abstract}
We compute four-loop corrections to the Higgs boson gluon vertex, including finite top quark mass effects. Analytic results are presented which serve as a building block for the next-to-next-to-next-toleading order corrections to Higgs boson production at the Large Hadron Collider at CERN.
\end{abstract}

DOI: $10.1103 /$ PhysRevLett.124.112002

Introduction.-The precise measurement of the standard model Higgs boson properties is a major focus of the physics program of the Large Hadron Collider (LHC) at CERN. A crucial quantity in this context is the total cross section for Higgs boson production in proton-proton collisions. There are several mechanisms which contribute to the cross section. The largest contribution is from the gluon-gluon fusion process [1] despite it being loop induced. It is thus important to have precise control over its higher-order quantum corrections.

Leading order (LO) corrections to the process $g g \rightarrow H$ were considered 40 years ago [2] and next-to-leading order (NLO) corrections were computed at the beginning of the nineties, first in the infinite top quark mass approximation [3,4] and shortly after exactly in $m_{t}$ [5]. (The analytic twoloop virtual corrections are known from [6-8].) About ten years later the first next-to-next-to-leading order (NNLO) results became available [9-11] in an effective-theory framework where the top quark is integrated out. It took a further ten years to compute corrections in $m_{H} / m_{t}$ and estimate the finite- $m_{t}$ effects. Several expansion terms of the Higgs-gluon form factor were computed in $[12,13]$ and subsequently an approximation method was developed to treat also the real-radiation contribution [14-17]. Recently, three-loop corrections to the Higgs-gluon vertex with a massive quark loop have been obtained by combining information from the large- $m_{t}$ and threshold expansions with the help of a conformal mapping and a Padé approximation [18]. For the subset of three-loop diagrams which contain a closed light-quark loop, even analytic results are available [19]. The next-to-next-to-next-to-leading order $\left(\mathrm{N}^{3} \mathrm{LO}\right)$ corrections to $g g \rightarrow H$ within the

Published by the American Physical Society under the terms of the Creative Commons Attribution 4.0 International license. Further distribution of this work must maintain attribution to the author(s) and the published article's title, journal citation, and DOI. Funded by SCOAP ${ }^{3}$. effective-theory approach have been computed in Refs. [20,21]. At this order in perturbation theory no finite- $m_{t}$ corrections are available. In this Letter we provide the first step to close this gap and compute the finite- $m_{t}$ effects of the virtual $\mathrm{N}^{3} \mathrm{LO}$ corrections. Note that the $g g \rightarrow H$ vertex diagrams only depend on the Higgs boson and top quark masses. Thus, it is promising to consider an expansion for large $m_{t}$ which is expected to show good convergence properties since the expansion parameter $\rho=m_{H}^{2} / m_{t}^{2} \approx 0.5$ is sufficiently small. Both at NLO and NNLO it has been shown that three expansion terms are adequate to obtain results which, from the phenomenological point of view, are equivalent to an exact calculation $[12,13]$.

In this work we concentrate on the numerically dominant contributions from diagrams in which the Higgs couples to a top quark loop. Note that although the Yukawa coupling is small, diagrams in which the Higgs couples to a bottom quark loop are parametrically enhanced by large logarithms. For example, the LO contribution is proportional to $m_{b}^{2} / m_{H}^{2} \log ^{2}\left(m_{b} / m_{H}\right)$ and thus bottom quark corrections should be included at lower orders.

Calculation.-The LO contribution to Higgs boson production in gluon fusion is mediated by the one-loop diagram shown in Fig. 1. Correspondingly $\mathrm{N}^{k} \mathrm{LO}$ corrections are obtained from $(k+1)$-loop vertex corrections also shown in Fig. 1. In this Letter, we compute the four-loop corrections.

The amplitude for $g g \rightarrow H$ can be parametrized as

$$
\mathcal{A}=\mathcal{A}_{0} h(\rho) \delta^{a b}\left(q_{1} \cdot q_{2} g^{\mu \nu}-q_{1}^{\nu} q_{2}^{\mu}\right)
$$

where $q_{1}$ and $q_{2}$ are the momenta of the external gluons with polarization vectors $\varepsilon^{\mu}\left(q_{1}\right)$ and $\varepsilon^{\nu}\left(q_{2}\right)$, respectively, and $a$ and $b$ are adjoint color indices. $\mathcal{A}_{0}$ consists of various coupling factors and mass parameters and is given by

$$
\mathcal{A}_{0}=T_{F} \frac{2 \alpha_{s}(\mu)}{3 v \pi},
$$



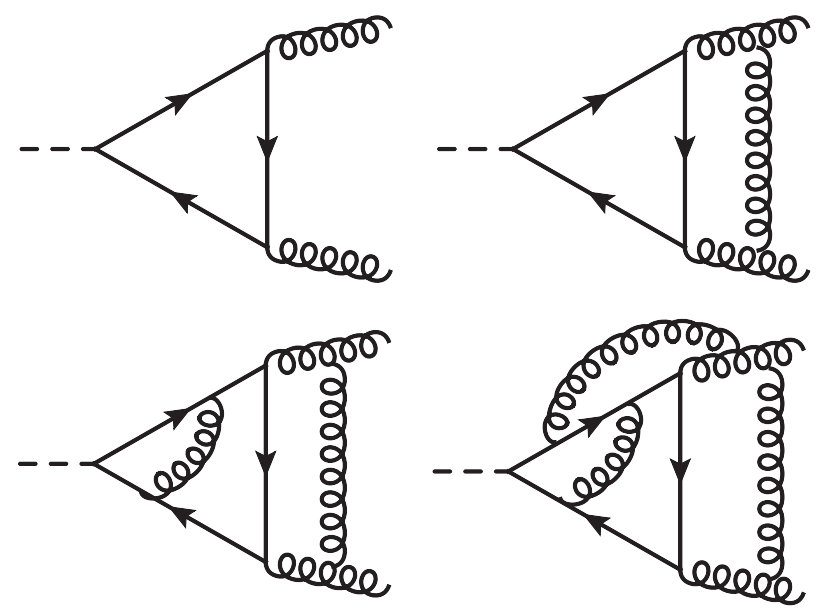

FIG. 1. Sample Feynman diagrams contributing to the process $g g \rightarrow H$ at LO, NLO, NNLO, and $\mathrm{N}^{3} \mathrm{LO}$. The solid and curly lines represent top quarks and gluons, respectively. The external dashed line stands for the Higgs boson.

where $T_{F}=1 / 2, v$ is the vacuum expectation value, and $\alpha_{s} \equiv \alpha_{s}^{(5)}$ is defined with five active quark flavors. The function $h(\rho)$ with $\rho=m_{H}^{2} / m_{t}^{2}$ contains the top quark mass dependence. It has the following expansion in the strong coupling constant:

$$
h(\rho)=\sum_{n \geq 1}\left(\frac{\alpha_{s}(\mu)}{\pi}\right)^{n-1} h^{(n)}(\rho),
$$

where the leading term is given by

$$
\begin{aligned}
h^{(1)}(\rho)= & \left(\frac{\mu^{2}}{m_{t}^{2}}\right)^{\epsilon} \Gamma(1+\epsilon)\left(1+\frac{7+7 \epsilon}{120} \rho\right. \\
& \left.+\frac{2+3 \epsilon+\epsilon^{2}}{336} \rho^{2}+\mathcal{O}\left(\rho^{3}\right)\right),
\end{aligned}
$$

with $\epsilon=(4-d) / 2$, where $d$ is the space-time dimension.

We apply projectors which independently project on the prefactors of $g^{\mu \nu}$ and $q_{1}^{\nu} q_{2}^{\mu}$ [cf. Eq. (1)] and treat the corresponding expressions independently. In the course of the calculation the intermediate expressions are different, however, the final results are equal (up to an overall sign) which is a welcome check for our calculation.

We apply an asymptotic expansion [22] in the limit $m_{t}^{2} \gg q_{1} \cdot q_{2}=m_{H}^{2} / 2$. This decomposes each Feynman diagram into a number of so-called "hard subgraphs" which have to be expanded in their external momenta. Afterwards, they appear as an effective vertex in the "cosubgraph" which is obtained from the original Feynman diagram after contracting all lines present in the hard subgraph to a point. From the technical point of view this leads to massive vacuum integrals up to four-loop order and massless vertex integrals, up to three-loop order, where only one external leg is off shell. Both types of integrals have been studied in the literature [23-31].

We organize our calculation such that the vacuum integrals are computed first. This requires that we solve tensor integrals since the integrand in general contains scalar products between the loop momenta of the vacuum integral and $q_{1}$ or $q_{2}$ or the loop momenta of the subsequent massless integration. Up to two-loop order there are general algorithms which treat tensor integrals of an arbitrary rank [32]. At three and four loops we have implemented tensor integrals up to rank eight, which is sufficient to obtain expansion terms up to $\rho^{2}$.

The application of the asymptotic expansion leads to a separation of the scales, at the price that the number of integrals to be computed is increased drastically; we have to consider around $40 \times 10^{6}$ three- and four-loop vacuum integrals and $1 \times 10^{6}$ one- to three-loop massless formfactor integrals. Because of the expansions in external momenta, many of the propagators are raised to relatively high powers. Similarly, the massless vertex integrations involve integrals with high powers of numerators.

We perform the reductions to master integrals with the help of FIRE [33] and use symmetry relations from LiteRed [34]. The combined size of the integral tables (as FORM tablebases) is about 25 GB. All master integrals are available in analytic form, both for the vacuum integrals [26] and the massless vertices [29].

The diagrams are computed by TFORM [35] jobs, each using four workers and requiring $20 \mathrm{~GB}$ of memory. The total wall time required by these jobs to compute the $\rho^{0}, \rho^{1}$, and $\rho^{2}$ terms of the expansion is approximately 6,50 , and 860 days, respectively.

The renormalization of the ultraviolet (UV) poles is straightforward. We first renormalize $\alpha_{s}$ and $m_{t}$ in the $\overline{\mathrm{MS}}$ scheme and the external gluon fields in the on-shell scheme. We then transform the $\overline{\mathrm{MS}}$ mass to the on-shell scheme and decouple the top quark from the running of $\alpha_{s}$; our final result is expressed in terms of $\alpha_{s}^{(5)}$. Note that one has to carefully include higher-order $\epsilon$ terms in the on-shell counterterms and the decoupling relations (see, e.g., Refs. [36,37]) since our final result still contains infrared poles.

There are several checks of our final result. First, we project on both structures present in Eq. (1) and check that they are equal up to a global sign. Then, we construct the leading $\left(\rho^{0}\right)$ term independently with the help of the effective-theory approach, i.e., we use the effective gluonHiggs coupling up to four loops [37-40] and the form factor results from the literature [27,30] to obtain the amplitude $\mathcal{A}$. Furthermore, the remaining poles after UV renormalization agree with the predictions provided, e.g., in Ref. [30,41]. Our three-loop results for the top quark mass corrections to the form factor agree with Refs. $[12,13]$.

When discussing the structure of the final result it is advantageous to extract the LO contribution and define 


$$
F=\frac{h(\rho)}{h^{(1)}(\rho)}=1+\mathcal{O}\left(\alpha_{s}\right)
$$

where an expansion in $\rho$ and in $\epsilon$ on the right-hand side is understood. Next, we consider $\log (F)$ since for this quantity there are simple predictions for the remaining infrared poles [30,41]. We expect that the pole part of $\log (F)$ has no expansion in $\rho$ and that the poles are given by the massless three-loop Higgs-gluon form factor. Our explicit results confirm these expectations. We thus define

$$
\log (F)=\log (F)_{\text {poles }}+\log (F)_{\text {finite }}
$$

and find that $\log (F)_{\text {poles }}$ reproduces the results given in Sec. 6 of Ref. [30]. $\log (F)_{\text {finite }}$ has an expansion in $\rho$ which we discuss in the remainder of this Letter.

Results.-We perform our calculation using general color structures for the gauge group $\mathrm{SU}(N)$. In order to present a compact expression we specify the color factors in the following to their numerical values for QCD. General expressions, for both the $\overline{\mathrm{MS}}$ and on-shell top quark mass, can be found in the supplementary material [42]. For $\mu^{2}=m_{t}^{2}$ we have for $\log (F)_{\text {finite }}$ in the on-shell scheme

$$
\begin{aligned}
& \log (F)_{\text {finite }}=+\frac{\alpha_{s}}{\pi}\left(\frac{11}{4}+\frac{1}{8} \pi^{2}-\frac{3}{4} l_{t H}^{2}+\frac{17}{135} \rho+\frac{3553}{226800} \rho^{2}\right) \\
& +\left(\frac{\alpha_{s}}{\pi}\right)^{2}\left[\frac{523}{108}+\frac{151}{192} \pi^{2}-\frac{499}{48} \zeta_{3}+l_{t H}\left(-\frac{155}{36}+\frac{23}{48} \pi^{2}+\frac{9}{8} \zeta_{3}\right)+l_{t H}^{2}\left(-\frac{151}{48}+\frac{3}{16} \pi^{2}\right)-\frac{23}{48} l_{t H}^{3}\right. \\
& +\rho\left(-\frac{15765509}{829440}+\frac{7}{1080} \pi^{2}+\frac{7}{540} \log (2) \pi^{2}+\frac{1909181}{110592} \zeta_{3}+\frac{793}{10368} l_{t H}\right) \\
& \left.+\rho^{2}\left(-\frac{1013177390077}{234101145600}+\frac{857}{907200} \pi^{2}+\frac{857}{453600} \log (2) \pi^{2}+\frac{267179777}{70778880} \zeta_{3}+\frac{580759}{43545600} l_{t H}\right)\right] \\
& +\left(\frac{\alpha_{s}}{\pi}\right)^{3}\left[-\frac{18539405}{1119744}+\frac{441517}{62208} \pi^{2}-\frac{11549467}{82944} \zeta_{3}-\frac{50839}{311040} \pi^{4}-\frac{1949}{576} \pi^{2} \zeta_{3}+\frac{39307}{288} \zeta_{5}\right. \\
& -\frac{39}{8} \zeta_{3}^{2}-\frac{193}{7560} \pi^{6}+l_{t H}\left(-\frac{322955}{31104}+\frac{665}{96} \pi^{2}-\frac{3043}{144} \zeta_{3}-\frac{1801}{5760} \pi^{4}-\frac{15}{16} \pi^{2} \zeta_{3}-\frac{27}{4} \zeta_{5}\right) \\
& +l_{t H}^{2}\left(-\frac{58745}{3456}+\frac{1435}{576} \pi^{2}+\frac{25}{8} \zeta_{3}-\frac{33}{320} \pi^{4}\right)+l_{t H}^{3}\left(-\frac{3995}{864}+\frac{23}{96} \pi^{2}\right)-\frac{529}{1152} l_{t H}^{4} \\
& +\rho\left(-\frac{542872693595}{3218890752}+\frac{65743583}{55987200} \pi^{2}-\frac{4691}{9720} \log (2) \pi^{2}-\frac{6788585826089}{107296358400} \zeta_{3}\right. \\
& -\frac{11421210133}{1149603840} \log ^{4}(2)+\frac{11364084757}{1149603840} \log ^{2}(2) \pi^{2}+\frac{244657561171}{55180984320} \pi^{4}-\frac{11421210133}{47900160} \mathrm{Li}_{4}(1 / 2) \\
& +\frac{718337}{9979200} \log ^{5}(2)-\frac{718337}{5987520} \log ^{3}(2) \pi^{2}+\frac{46111267}{239500800} \log (2) \pi^{4}-\frac{10073}{25920} \pi^{2} \zeta_{3}-\frac{3254515597}{31933440} \zeta_{5} \\
& \left.-\frac{718337}{83160} \mathrm{Li}_{5}(1 / 2)+\frac{5327119}{11197440} l_{t H}+\frac{25639}{746496} l_{t H}^{2}\right) \\
& +\rho^{2}\left(-\frac{1055794361417882487061}{6681366555210547200}+\frac{4077367559}{23514624000} \pi^{2}+\frac{23157917500539717053}{117837152649216000} \zeta_{3}\right. \\
& -\frac{110153}{1632960} \log (2) \pi^{2}+\frac{2712037738087}{2391175987200} \log ^{4}(2)-\frac{2729355664999}{2391175987200} \log ^{2}(2) \pi^{2}-\frac{150868470717581}{229552894771200} \pi^{4} \\
& +\frac{2712037738087}{99632332800} \mathrm{Li}_{4}(1 / 2)+\frac{46902913}{202176000} \log ^{5}(2)-\frac{46902913}{121305600} \log ^{3}(2) \pi^{2}-\frac{8632107859}{33965568000} \log (2) \pi^{4} \\
& \left.\left.-\frac{1233223}{21772800} \pi^{2} \zeta_{3}+\frac{49563452909}{4528742400} \zeta_{5}-\frac{46902913}{1684800} \mathrm{Li}_{5}(1 / 2)+\frac{103150403081}{658409472000} l_{t H}+\frac{18740929}{3135283200} l_{t H}^{2}\right)\right] \text {, }
\end{aligned}
$$


where $\zeta_{n}$ is the Riemann $\zeta$ function evaluated at $n, \mathrm{Li}_{n}$ denote polylogarithms, $l_{t H}=\log \left(m_{t}^{2} / m_{H}^{2}\right)+i \pi$ and $m_{t}$ is the top quark pole mass. It is interesting to compare the finite $m_{t}$ corrections of $\log (F)_{\text {finite }}$ for the various orders is $\alpha_{s}$. The numerical evaluation of Eq. (7) for $m_{t}=173 \mathrm{GeV}$ and $m_{H}=125 \mathrm{GeV}$ gives

$$
\begin{aligned}
\log (F)_{\text {finite }} \approx & a_{t}[(11.07-i 3.06)+0.07+0.004] \\
& +a_{t}^{2}[(22.59+i 13.24)+(1.02+i 0.13) \\
& +(0.07+i 0.01)] \\
& +a_{t}^{3}[(-73.18+i 51.55)+(7.61+i 0.85) \\
& +(0.70+i 0.14)],
\end{aligned}
$$

where $a_{t}=\alpha_{s}^{(5)}\left(m_{t}\right) / \pi$. For each order in $a_{t}$ we separately display the $\rho^{0}, \rho^{1}$, and $\rho^{2}$ terms inside the square brackets. One observes that the mass corrections become more important when going to higher orders in $a_{t}$. At two loops the $\rho^{1}$ contribution only amounts to $0.6 \%$ of the real part of the leading term, whereas at three and four loops we have real contributions of $5 \%$ and $10 \%$, respectively. At fourloop order the $\rho^{2}$ real contributions are below $1 \%$ which justifies the truncation of the expansion at this order; we expect that the next term is negligibly small. In all cases the imaginary parts converge at least as well as the real parts.

If we repeat the same exercise for the $\overline{\mathrm{MS}}$ top quark mass, $\bar{m}_{t}$, and set the renormalization scale to $\mu^{2}=\bar{m}_{t}^{2}$, we observe smaller mass corrections; at one, two, and three loops the $\rho^{1}$ terms contribute $0.1 \%, 2.5 \%$, and $1.4 \%$, respectively, relative to the real part of the $\rho^{0}$ terms. In all cases the $\rho^{2}$ terms are smaller again by a factor of 5 to 10 .

For Higgs boson production the central value of the renormalization scale is often set to $\mu^{2}=m_{H}^{2} / 2$. Adopting the on-shell scheme for the top quark mass, this leads to $\rho^{1}$ real contributions which amount to $0.5 \%, 34 \%$, and $1.8 \%$ for two, three, and four loops. Note that the large relative correction at three loops is due to accidental cancellations which make the leading term $\left(\rho^{0}\right)$ quite small at this order.

Conclusions. - In this Letter, we compute finite top quark mass effects for the production virtual cross section of the standard model Higgs boson. We expand the fourloop Higgs-gluon vertex diagrams for $m_{t} \gg m_{H}$ and show that three expansion terms are sufficient to obtain precision results for the physical values of $m_{H}$ and $m_{t}$. Our result is the first $\mathrm{N}^{3} \mathrm{LO}$ calculation of the Higgs production cross section which incorporates finite top quark mass terms. In the coming years, the LHC enters the era of precision Higgs boson physics and quantum corrections such as those computed in this Letter will become important.

This research was supported by the Deutsche Forschungsgemeinschaft (DFG, German Research Foundation) under Grant No. 396021762-TRR 257
"Particle Physics Phenomenology after the Higgs Discovery." F. H. acknowledges the support of the DFGfunded Doctoral School KSETA.

*joshua.davies@kit.edu

florian.herren@kit.edu

*matthias.steinhauser@kit.edu

[1] D. de Florian et al. (LHC Higgs Cross Section Working Group), arXiv:1610.07922.

[2] H. M. Georgi, S. L. Glashow, M. E. Machacek, and D. V. Nanopoulos, Phys. Rev. Lett. 40, 692 (1978).

[3] A. Djouadi, M. Spira, and P. M. Zerwas, Phys. Lett. B 264, 440 (1991).

[4] S. Dawson, Nucl. Phys. B359, 283 (1991).

[5] M. Spira, A. Djouadi, D. Graudenz, and P. M. Zerwas, Nucl. Phys. B453, 17 (1995).

[6] R. Harlander and P. Kant, J. High Energy Phys. 12 (2005) 015 .

[7] C. Anastasiou, S. Beerli, S. Bucherer, A. Daleo, and Z. Kunszt, J. High Energy Phys. 01 (2007) 082.

[8] U. Aglietti, R. Bonciani, G. Degrassi, and A. Vicini, J. High Energy Phys. 01 (2007) 021.

[9] R. V. Harlander and W. B. Kilgore, Phys. Rev. Lett. 88, 201801 (2002).

[10] C. Anastasiou and K. Melnikov, Nucl. Phys. B646, 220 (2002).

[11] V. Ravindran, J. Smith, and W. L. van Neerven, Nucl. Phys. B665, 325 (2003).

[12] R. V. Harlander and K. J. Ozeren, Phys. Lett. B 679, 467 (2009).

[13] A. Pak, M. Rogal, and M. Steinhauser, Phys. Lett. B 679, 473 (2009).

[14] R. V. Harlander and K. J. Ozeren, J. High Energy Phys. 11 (2009) 088.

[15] A. Pak, M. Rogal, and M. Steinhauser, J. High Energy Phys. 02 (2010) 025.

[16] R. V. Harlander, H. Mantler, S. Marzani, and K. J. Ozeren, Eur. Phys. J. C 66, 359 (2010).

[17] A. Pak, M. Rogal, and M. Steinhauser, J. High Energy Phys. 09 (2011) 088.

[18] J. Davies, R. Gröber, A. Maier, T. Rauh, and M. Steinhauser, Phys. Rev. D 100, 034017 (2019).

[19] R. V. Harlander, M. Prausa, and J. Usovitsch, J. High Energy Phys. 10 (2019) 148.

[20] C. Anastasiou, C. Duhr, F. Dulat, E. Furlan, T. Gehrmann, F. Herzog, A. Lazopoulos, and B. Mistlberger, J. High Energy Phys. 05 (2016) 058.

[21] B. Mistlberger, J. High Energy Phys. 05 (2018) 028.

[22] V. A. Smirnov, Springer Tracts Mod. Phys. 250, 1 (2012).

[23] S. Laporta, Phys. Lett. B 549, 115 (2002).

[24] Y. Schroder and A. Vuorinen, J. High Energy Phys. 06 (2005) 051.

[25] K. G. Chetyrkin, M. Faisst, C. Sturm, and M. Tentyukov, Nucl. Phys. B742, 208 (2006).

[26] R. N. Lee and I. S. Terekhov, J. High Energy Phys. 01 (2011) 068.

[27] P. A. Baikov, K. G. Chetyrkin, A. V. Smirnov, V. A. Smirnov, and M. Steinhauser, Phys. Rev. Lett. 102, 212002 (2009). 
[28] G. Heinrich, T. Huber, D. A. Kosower, and V. A. Smirnov, Phys. Lett. B 678, 359 (2009).

[29] R. N. Lee and V. A. Smirnov, J. High Energy Phys. 02 (2011) 102.

[30] T. Gehrmann, E. W. N. Glover, T. Huber, N. Ikizlerli, and C. Studerus, J. High Energy Phys. 06 (2010) 094.

[31] T. Gehrmann, E. W. N. Glover, T. Huber, N. Ikizlerli, and C. Studerus, J. High Energy Phys. 11 (2010) 102.

[32] K. G. Chetyrkin, in Oberammergau 1993, New Computing Techniques in Physics Research III (Proceedings of AIHENP-93) (World Scientific, Singapore, 1994), pp. 559563 [arXiv:hep-ph/0212040].

[33] A. V. Smirnov and F. S. Chuharev, arXiv:1901.07808.

[34] R. N. Lee, J. Phys. Conf. Ser. 523, 012059 (2014).
[35] B. Ruijl, T. Ueda, and J. Vermaseren, arXiv:1707.06453.

[36] P. Marquard, A. V. Smirnov, V. A. Smirnov, M. Steinhauser, and D. Wellmann, Phys. Rev. D 94, 074025 (2016).

[37] M. Gerlach, F. Herren, and M. Steinhauser, J. High Energy Phys. 11 (2018) 141.

[38] K. G. Chetyrkin, B. A. Kniehl, and M. Steinhauser, Nucl. Phys. B510, 61 (1998).

[39] K. G. Chetyrkin, J. H. Kühn, and C. Sturm, Nucl. Phys. B744, 121 (2006).

[40] Y. Schroder and M. Steinhauser, J. High Energy Phys. 01 (2006) 051.

[41] T. Becher and M. Neubert, Phys. Rev. Lett. 102, 162001 (2009); 111, 199905(E) (2013).

[42] https://www.ttp.kit.edu/preprints/2019/ttp19-039/. 